\title{
非肌层浸润性膀胱癌经尿道电切术治疗效果、复发情况 的研究
}

\author{
王 博* 李炳义 \\ 渭南市第二医院，陕西 714000
}

摘 要: 目的: 研究经尿道电切术 ( TURBT) 治疗非肌层浸润性膀胱癌 (NMIBC) 的临床效果。方法: 选取 2017年8月至2019年1月在我院治疗的94例 NMIBC患者作为研究对象, 依据简单随机化法将其分为观察组和对照组, 各 47例。对照组实施开放性手术治疗, 观察组实施TURBT治疗, 比较两组手术治疗效果。结果：观察组的各项手术指标 均优对照组; 观察组术后的KPS评分高于对照组; 并发症发生率均于对照组, $P<0.05$; 两组复发率相比, $P>0.05$ 。 结论：TURBT在治疗NMIBC中可优化围术期各项指标，提高预后生存质量，降低并发症和复发率。

关键词：经尿道电切术；非肌层；浸润性膀胱癌；围术期指标

\section{一、前言}

膀胱癌是起源于膀胱的恶性肿瘤, 此病的发生和环境、吸烟有关, 膀胼癌可发生在任何年龄中, 以中老年男性人 群居多, 发病率随着年龄的增长而增加, 根据治疗模式和疾病预后的不同可分为肌层浸润性膀胱癌（MIBC）以及非 肌层浸润性膀胱癌（NMIBC），此病的发病原因至今尚不清楚，可能存在遗传因素和外在环境因素共同影响所致 ${ }^{[1]}$ 。 无痛血尿、尿频、尿急、尿痛、排尿困难是此病的临床症状, 患者常以肉眼可见的血尿而就诊。临床上治疗NMIBC 主要以外科手术为主, 手术会对患者带来一定创伤, 术后也容易发生各类并发症 ${ }^{[2]}$ 。随着微创的介人, 经尿道电切术 创伤更小、术后恢复快、并发症少, 受到临床医师的青睐。本文就TURBT术应用在NMIBC患者中的手术效果进行研 究，现报告如下。

\section{二、资料及方法}

\section{(一) 一般资料}

选取2017年8月至2019年1月在我院治疗的94例NMIBC患者参与本次研究, 采用简单随机化法将上述患者分成观察组 和对照组, 各47例。观察组: 男性31例、女性16例, 年龄42 69岁、平均 (54.35 44.68) 岁; 对照组: 男性30例、女性 17 例，年龄43 70岁、平均 (54.37 \pm 4.72$)$ 岁，对比分析两组的一般资料，无统计学差异 $(P>0.05)$ ，具有可比性。

纳人标准: 所有患者均为非肌层浸润性膀胱癌患者; 所有患者及其家属对本次研究知情并签署知情同意书; 随 访 $\geqslant 1$ 年; 本研究经本院伦理委员会审核并批准。排除标准: 无法耐受手术者; 伴有其他恶性肿瘤疾病; 膀胱外浸润 者; 资料不全者。

(二) 方法

对照组采取开放性膀胱切除术, 方法: 取患者仰卧位, 麻醉后, 于下腹部正中线作 $15 \mathrm{~cm}$ 切口, 行膀胱全切除 术, 并全面清扫整个盆腔淋巴结, 行输尿管皮肤造口术, 术毕, 缝合创口, 术后常规抗感染治疗。观察组采取经尿道 电切术, 方法: 做好术前准备, 取患者截石位, 麻醉后, 探查肿瘤情况, 直视下置人电切镜, 对小的肿瘤从肿瘤基底 部膀胱壁进行电切直至深肌层, 并对肿瘤边缘 $2 \mathrm{~cm}$ 处快速清除, 对较大的肿瘤从肿瘤顶部进行电切直至将脂肪层充分 暴露, 采用电凝止血, 行电切操作时确保膀胱处于半充盈或者充盈状态, 保证术野清晰, 术后放置三腔气囊导尿管, 观察引流液颜色, 做好尿道口维护, 术后4 5 d拔出导尿管, 术后6周每次给予 $9 \mathrm{~Gy}$ 的放射药物治疗, 总量 $54 \mathrm{~Gy}$, 放疗 的过程使用吉西他滨结合顺铂联合化疗3周。

\section{(三) 观察指标}

对比两组的相关手术指标如（手术、住院、导尿管留置）时间、术中出血量；对比两组治疗前后的KPS评分；统

“通讯作者: 王博, 1981年11月, 男, 汉族, 陕西渭南人, 现任职于渭南市第二医院泌尿外科, 主治医师, 本 科。研究方向: 前列腺增生激光剜除及前列腺肿瘤的根治。 
计两组术后的并发症发生率以及随访 1 年时间的疾病复发率。

( 四) 评价标准

采用Karnofsky功能状态评分标准 (KPS) 对两组治疗前后的健康状态进行评分, KPS评分 $\geqslant 80$ 表示可生理自理; KPS分数50 70分表示生活半自理; KPS评分 $<50$ 分表示生活需要他人帮助, 80 分以上则术后状态很好, 存活期较长 ${ }^{[3]}$ 。

(五) 统计学分析

采用统计学软件SPSS21.0处理研究数据, 计量资料: “ “ $\bar{x} \pm s$ ”, $t$ 检验; 计数资料: $(n, \%), \chi^{2}$ 检验。 $P<$ 0.05: 差异有统计学意义。

\section{三、结果}

(一) 两组手术相关指标比较

两组手术相关指标相比，差异具备统计学意义 $(P<0.05)$, 如表1所示。

表1 两组手术指标对比表 $(\overline{\boldsymbol{x}} \pm s)$

\begin{tabular}{cccccc}
\hline 组别 & 例数 & 手术时间 $(\mathrm{min})$ & 术中出血量 $(\mathrm{mL})$ & 导尿管留置时间 $(\mathrm{d})$ & 住院时间 $(\mathrm{d})$ \\
\hline 观察组 & 47 & $41.36 \pm 2.27$ & $42.57 \pm 3.36$ & $6.17 \pm 1.38$ & $8.17 \pm 1.32$ \\
对照组 & 47 & $91.38 \pm 3.24$ & $85.32 \pm 4.26$ & $9.39 \pm 1.42$ & $13.64 \pm 2.35$ \\
$t$ & - & 86.682 & 54.018 & 11.149 & 13.913 \\
$P$ & - & $<0.05$ & $<0.05$ & $<0.05$ & $<0.05$ \\
\hline
\end{tabular}

(二) 两组治疗前后的KPS评分对比

术前两组KPS评分相比, 无统计学差异 $(P>0.05)$; 术后两组的KPS评分均有所升高, 观察组的高于对照组, 比 较差异具备统计学意义 $(P<0.05)$, 如表2所示。

表2 两组KPS评分对比表 $(\bar{x} \pm s$, 分 $)$

\begin{tabular}{cccccc}
\hline 组别 & 例数 & 治疗前 & 治疗后 & $t$ & $P$ \\
\hline 观察组 & 47 & $61.35 \pm 8.64$ & $88.75 \pm 6.49$ & 17.383 & $<0.05$ \\
对照组 & 47 & $62.45 \pm 9.36$ & $72.45 \pm 5.73$ & 6.247 & $<0.05$ \\
$t$ & - & 0.592 & 12.907 & - & - \\
$P$ & - & $>0.05$ & $<0.05$ & - & - \\
\hline
\end{tabular}

(三) 两组并发症发生率对比

观察组的并发症发生率为 $6.38 \%$; 对照组的为 $23.40 \%$ ，有显著统计学差异， $P<0.05$, 如表3所示：

表3 两组并发症发生率对比表 $[n(\%)]$

\begin{tabular}{cccccc}
\hline 组别 & 例数 & 膀胱痉挛 & 闭孔反射 & 膀胱穿孔 & 总发生率 \\
\hline 观察组 & 47 & $3(6.38)$ & $0(0.00)$ & $0(0.00)$ & $3(6.38)$ \\
对照组 & 47 & $4(8.51)$ & $5(10.64)$ & $2(4.26)$ & $11(23.40)$ \\
$\chi^{2}$ & - & - & - & - & 5.371 \\
$P$ & - & - & - & - & 0.020 \\
\hline
\end{tabular}

( 四) 两组复发率对比

随访 1 年, 观察组的复发例数为 4 例、复发率为 $8.51 \%(4 / 47)$; 对照组复发例数为 3 例、复发率为 $6.38 \%$ (3/47) , $\chi^{2}=0.154, P=0.694$ 。

四、讨论

膀胱癌是泌尿外科常见的恶性肿瘤, 是泌尿系统中尿路转移到上皮的恶性肿瘤, 疾病发生的原因主要是尿路上皮 细胞受到刺激而引起的基因分子水平突变, 如长期受到各种刺激如化学、物理、生物等, 而原致癌基因的激活在复 制、转录等过程中未及时修复受损的DNA, 导致细胞周期的改变, 使癌细胞无限制的增殖而发生癌变。我国男性膀胱 癌的发病率在高发性肿瘤中排行第七, 女性发病率更低, 男性吸烟、激素分泌、压力是导致男性发病率高于女性的主 要原因 ${ }^{[4]}$ 。

临床治疗NMIBC可采取全膀胱切除术、部分膀胱切除术, 这种治疗方式对NMIBC有很好的控制作用, 但是术中 
出血量较大、创伤也大、并发症也多, 况且大部分患者年龄较大, 术后恢复慢, 对其生存质量带来很大影响。随着腔 镜技术的快速发展 ${ }^{[5]}$, TURBT也在治疗不同泌尿系统疾病中得到了广泛的应用并取得了良好的治疗效果, 该治疗手段 对机体的创伤比较小, 患者容易接受, 手术也相对比较安全, 术后不影响患者的正常排尿功能 ${ }^{[6]}$ 。激光和外切不同的 能量形式, 而操作方法相似, 这种特定波长激光在水中传播过程, 能量不流失, 能被血红蛋白选择性吸收, 可达到良 好的凝固和阻断血管的作用, 也有良好的止血效果, 留置尿管时间也会缩短, 而且激光术视野更清楚, 对病灶的定位 更加精准，有高效的组织汽化、凝固效果，同时又具备热量组织穿透浅的特点，不容易对周围组织造成损伤，降低了 术中发生膀胱穿孔的风险 ${ }^{[7]}$ 。这种手术方法可通过激光能量切割组织, 并非真正的接触组织, 术中无电流通过组织, 不会对膀胱壁神经造成刺激, 完全消除了发生闭孔神经反射的风险, 由于无电流干扰, 对一些装有心脏起搏器的患者 也非常安全, 激光通过汽化凝固可更好的封闭淋巴管及毛细血管, 减少了肿瘤细胞的传播 ${ }^{[8]}$ 。本研究结果显示, 观察 组围手术期的各项指标也优于对照组; 治疗后的KPS生存质量评分高于对照组, 术后并发症、复发率均低于对照组, 可见TURBT对NMIBC的治疗可取得良好的临床效果，实际运用时还需联合放化疗治疗，降低复发率。

\section{五、结论}

综上所述, TURBT应用在NMIBC的治疗中, 不仅具有和膀胱切除术类似的治疗效果, 围术期安全性更好, 术后 生存质量也更高，术后并发症发生率和疾病复发率也比较低，值得进一步推广研究。

\section{参考文献:}

[1]石昌龙, 宋永胜.经尿道同期电切膀胱肿瘤和良性前列腺增生组织患者的安全性和生活质量分析 [J].现代肿瘤医学, 2020,28(10):1715-1718.

[2]陈果, 吴波, 陈亮.同期经尿道电切术联合坦索罗辛治疗膀胱肿瘤合并前列腺增生患者的临床研究 [J]. 中国处方药, 2019,17(12):130-131.

[3]刘杨. 经尿道电切术治疗非肌层浸润性膀胱癌的效果及复发率分析[J].吉林医学, 2019,40(12):2838-2839.

[4]陈志华, 帅强, 廖俩娣. 经尿道电切术联合放化疗治疗肌层浸润性膀胱癌的效果及复发率分析 [J]. 齐齐哈尔医学院学 报, 2019,40(22):2849-2850.

[5]H.Yan,X.Zhou,X.Y.Wang,R.Li,Y.P.Shi,Q.Xia, 戚建晨. 延迟 (18)F-FDG PET/CT成像在经尿道膀胱癌切除术后残余 肿瘤评估中的应用 [J].国际医学放射学杂志, 2019,42(06):738.

[6]李文㻇,刘明建, 张启飞, 梁威飘, 吴保忠, 杨帝宽, 张伟健. 超选择动脉栓塞联合经尿道电切术治疗高危膀胱癌的临床 研究 [J].临床医学工程, 2019,26(11):1507-1508.

[7]梁昌景, 潘建海, 吴扬, 陈森.经尿道钬激光膀胱肿瘤切除术与经尿道膀胱肿瘤电切术治疗表浅层膀脱癌的效果对比 [J]. 中国当代医药, 2018,25(35):59-61.

[8]韩红举.经尿道膀胱瘤电切术加药灌注治疗浅表性膀胱瘁的临床疗效观察[J].中国医药科学, 2018,8(22):216-219. 